J urnal Teknologi, 44(B) J un 2006: 1-15

(C) Universiti Teknologi Malaysia

\title{
THE USE OF PHOTOGRAMMETRY TECH NIQUES TO EVALUATE THE CONSTRUCTION PROJECT PROGRESS
}

\author{
ZUBAIR AHMED MEMON ${ }^{1}$, MUHD ZAIMI ABD. MAJID $^{2} \&$ \\ MUSHAIRRY MUSTAFFAR ${ }^{3}$
}

\begin{abstract}
The modeling of 3D objects from image sequence is a challenging problem and has been an important research topic in the areas of photogrammetry and computer vision for many years. Photogrammetry is the science of calculating 3D object co-ordinates from image and provides a flexible and robust approach for measuring the static and dynamic characteristics for construction management. This paper discusses the experience in Construction Technology and Management Centre (CTMC), Universiti Teknologi Malaysia (UTM) in adapting photogrammetry methods for specific problems in the construction industry and outlines the principles of close-range photogrammetry in evaluating the progress of construction projects. There is a need to use the principles of close-range photogrammetry to evaluate the progress of construction project and to develop the actual progress bar chart. The fundamental task of photogrammetry is to rigorously establish the geometric relationship between the image and the object, as it existed at the time of imaging event. One such software application is PhotoModeler Pro version from the Canadian company Eos System has been suggested to extract the 3D features from 2D images. The approach described in this paper demonstrates the use of digital photogrammetry as a complementary method, which describes the 3D features extraction procedure in detail and highlights the qualitative control that can be achieved during the construction. The technique uses mainly off-the-shelf digitalcamera and software technologies that are affordable to most organisations and able to provide acceptable accuracy.
\end{abstract}

K eywords: Close-range photogrammetry, digital photographs, 3D-coordinates, software and digital monitoring system

\subsection{INTRODUCTION}

Construction process is a process during which construction products are being made and includes several sub-processes, which recursively include other subprocesses [1]. Construction projects during their accomplishment in the field require much time and expense, and close management control of them is

1 Construction Technology and Management Centre (CTMC), Block C-09, Faculty of Civil Engineering, Universiti Teknologi Malaysia (UTM), 81310 Skudai, J ohor, Malaysia Tel: +6017 712 5682, Fax: +607 553 1934, Email: zubairahmedmemon@yahoo.com or zubair@siswa.utm.my

2 Faculty of Civil Engineering, Universiti Teknologi Malaysia, 81310 Skudai, J ohor, Malaysia Tel: 07-5531935, Email: zaimi@fka.utm.my

3 CTMC, Faculty of Civil Engineering, Universiti Teknologi Malaysia, 81310 Skudai, Johor, Malaysia. Email: mustaffar@fka.utm.my 
required if they are to be completed within the established time, cost limitations and within prescribed quality. Project conditions such as technical complexity, importance of timely completion, resource limitations, and substantial costs put great emphasis on the planning, scheduling, and control of construction operations. Unfortunately, the construction process, once it is set into motion, is not a self-regulating mechanism and requires expert guidance if events are to conform to plans. In order to function professionally in today's construction project, one must be able to recognise and diagnose problems as they evolve and apply the appropriate principles in order to avoid or resolve these problems [2, 3].

Abd. Majid [4] mentioned that with the development of new scientific management concepts, comprehensive management techniques have been applied from inception to completion of construction operations. H owever, he also added that once the project started, monitoring systems are established that measure actual cost and progress of the work at periodic intervals and the reporting system provides progress information that is measured against the planned schedule of work. The resulting time schedule, subject to periodic revision and correction during construction, is the essential basis for the day-to-day time control of the project. Such a schedule serves as an exceptionally effective early-warning device for detecting when and where the project is falling behind.

$\mathrm{H}$ sieh [5] introduced Site-city, a semi automated building extraction system integrating photogrammetry, geometric constraints and image understanding algorithms. Site-city uses rigorous photogrammetric principles and multiple images to accurately determine 3D locations of objects, such as buildings or roads in the scene. Site city automated process affects a combination of scene complexity, image contrast, view angles, and user performance. Streilein [6] developed the system, which integrates the data and the CAAD-based 3D feature extraction. The dataintegration is achieved using a knowledge-base system and digital photogrammetry system (DI PS) was used for 3D feature extraction. This system combines digital photogrammetric methods with the capabilities of CAAD. Streilein [7] described an object-oriented approach using CAD models for the initialization of an automatic measurement process and embedded in the software environment with digital photogrammetry. Various examples on the use of CAD models in photogrammetry applications can be found in the fields of architecture, heritage recording and industrial applications.

Digital images are increasingly becoming more important in the photogrammetric field. Their primary advantage is to permit a higher degree of automation within evaluation processes of construction activities. The basic task of many photogrammetric systems is to derive object space coordinates from 2D images. Analog, semi analytical and analytical techniques have been employed for a long period of time in photogrammetry to extract ground coordinates of objects 
from hardcopy images. In recent years, digital techniques are implemented in photogrammetric applications. The advantages of using digital techniques are: the ease and speed of data acquisition, the inherent on-line and real-time capabilities, and the high degree of automation. Acquiring digital images is done either by scanning hardcopy images or by capturing the photographs directly in digital format using digital sensors. Gomes [8] presented the results of an architectural photogrammetric project using the PhotoModeler software. The results showed that with comparison to the conventional photogrammetric procedure, photomodeler allows even greater reduction of time and costs for the production of models, since it does not require positioning and measuring of targets and stereoscopy to produce suitable photographic documents.

Edward [9] described photogrammetry as the process of deriving (usually) metric information about an object through measurements made on photographs of the object. In addition to photographs, other types of images can also be used, such as those electronically captured on tape or from digital sources such as cameras or video recorders [10]. The classical application of photogrammetry known as topographic photogrammetry is for creating aerial surveys and maps. There are also many ground-based applications known as non-topographic or close-range photogrammetry in such diverse fields as archaeology, bio-engineering, civil engineering, computer animation, forensic analysis, historical preservation, mechanical inspection, plant engineering, ship construction and surgery [11]. Modern close-range photogrammetry uses digital imaging sensors and computer data analysis and often measures hundred or thousands of object points. The fundamental theory is based on surveying principles. When dealing with time sequences of images, the term videogrammetry or video-metrics is used to describe this technology. Photogrammetry offers the simplicity of taking photographs coupled with good to excellent measurement precision [12].

An integrated project progress monitoring and evaluation system called Automated Construction Project Monitoring (ACPROM ${ }^{\circledR}$ ) has been developed to assist project managers in developing the actual physical progress reports. ACPROM ${ }^{\circledR}$ calculates the percentage of progress by integrating the digital images and AutoCAD drawings and plots the actual progress bar chart. To develop the actual project progress reports coordinate values from 3D CAD drawings and 3D model from digital images are stored in the databases and the percentage of progress are calculated by simulating the databases. The ACPROM ${ }^{\circledR}$ will show the progress bar chart by comparison of as-planned schedule of work with as-built schedule of work. To develop the 3D model from digital images, an accurate analytical method is required for calculating 3D coordinates values, which will be used for calculating the progress of the work. This paper proposes the framework model and discusses initial experience at CTMC, UTM, by using photogrammetry techniques to evaluate the construction site progress. 


\subsection{AVAILABLE METHOD}

In general, photogrammetric projects can be broadly dassified as those requiring satellite imagery, those requiring airborne imagery, and close-range or industrial applications. The majority of photogrammetric operations have historically involved the use of aerial photographs. Close-range and industrial applications are characterized by having the cameras or sensors on very near the surface of the earth, usually stationary. Film-based, video, and digital cameras are all being used for a variety of applications.

A photogrammetric project involves two general functions [9]:

(i) Acquisition and preparation of imagery and support data.

(ii) Processing of the imagery to derive the required products.

The first function encompasses several operations, such as project definition, specifications, and planning; acquisition of suitable imagery; preprocessing of the imagery in preparation for use in the photogrammetric reduction or processing system; and collection of other, supporting data such as survey-derived ground control. The second function includes deciding which photogrammetric system to use based on the specified products expected from project.

\subsection{Interior Orientation}

The interior orientation defines the sensor or camera characteristic required for reconstruction of the object space bundle of rays from the corresponding images points. In a frame camera, these characteristics would include at least the focal length of principal distance, the location of the principal point in the image plane, and a description of the lens distortion.

\subsection{Exterior Orientation}

Interior orientation, establishes the bundle of rays from the image points. Exterior orientation or platform model, establishes the position and orientation of the bundle of rays or an image in space with respect to the object space coordinate system. Each bundle requires six independent elements; three for position and three for orientation. The parameters include the object space coordinates of the perspective center and three angles, $\kappa, \phi$, $\omega$, which describe the orientation of the object space coordinate system with respect to the image coordinate system. The calculation of the object space coordinates of a point from its coordinates in two or more images or intersection refers to the determination of a point's position in object space by intersecting the image rays from two or more images.

For a bundle of rays, the three elements of position fix the center of perspective and coordinates are often referred to as the camera station and expressed by [9] as, 


$$
L=\left[\begin{array}{c}
X_{L} \\
Y_{L} \\
Z_{L}
\end{array}\right]
$$

Analytical geometry tells us that three angles, or three independent parameters, are sufficient to describe the orientation or attitude of this bundle in the object space coordinate system. This is equivalent to saying that three independent parameters are necessary to define the rotation matrix that relates the object space and image space systems. The exterior orientation defines the relationship between the object and image space coordinates systems by the following equation: in this equation $(x, y,-f)$ are the image space coordinates, $k$ is a scale factor, $M$ is a $3 \times 3$ matrix containing the rotation parameters, and $(X, Y, Z)$ represent the object point.

$$
\left[\begin{array}{c}
x \\
y \\
-f
\end{array}\right]=k M\left[\begin{array}{c}
X-X_{L} \\
Y-Y_{L} \\
Z-Z_{L}
\end{array}\right]
$$

The standard approach to constructing $M$ is by using three sequential rotations: $\omega$ about the x-axis, $\phi$ about once-rotated the $y$-axis, $\kappa$ about the twice rotated z-axis. Edward [9] and Atkinson [11] constructed the total rotation matrix as: $M=$ $\mathrm{M} \kappa \mathrm{M} \phi \mathrm{M}_{\omega}$

$$
\left[\begin{array}{ccc}
\cos \phi \cdot \cos \kappa & \cos \omega \cdot \sin \kappa+\sin \omega \cdot \sin \phi \cdot \cos \kappa & \sin \omega \cdot \sin \kappa-\cos \omega \cdot \sin \phi \cdot \cos \kappa \\
-\cos \phi \cdot \sin \kappa & \cos \omega \cdot \cos \kappa+\sin \omega \cdot \sin \phi \cdot \sin \kappa & \sin \omega \cdot \cos \kappa-\cos \omega \cdot \sin \phi \cdot \sin \kappa \\
\sin \phi & -\sin \omega \cdot \sin \phi & \cos \omega \cdot \cos \phi
\end{array}\right]
$$

\subsection{Resection}

The basic operations of photogammetry are resection, the determination of an image's position and orientation parameters with respect to an object space coordinate system. In the standard case, the parameters include the object space coordinates of the perspective center and three angles, $\kappa, \phi$, $\omega$, which describe the orientation of the object space coordinate system with respect to the image coordinate system.

\subsection{Intersection}

Intersection refers to the determination of a point's position in object space by intersecting the image rays from two or more images. 


\subsection{CLOSE-RANGE PHOTOGRAMMETRY METHOD}

The term 'close-range photogrammetry' is used to describe the technique when the extent of the object to be measured is less than about 100 metres and cameras are positioned close to it [11]. In the past, close-range applications have been distinguishable from topographic applications by size, appearance and shape of the objects and by the size of the images. The differences for the objects will remain for the future but the size of the image data will get closer [13].

The result of close range photogrammetry must generally be made available very quickly after acquisition of the images so that they can be used for further processing related to the measured object and its function. Derived coordinates might be used for comparing the measured object with its designed size and shape in a test of conformance. They are sometimes processed further using computer graphics, for example to produce a three dimensional CAD model of the object and in a few cases drawn and dimensional plans, elevations or sections may be required.

The development of analytical photogrammetric techniques has enabled rapid growth in the applications of close-range photogrammetry. The ability to model the camera geometry mathematically, instead of relying on analog restitution, allows the use of a wide variety of non-metric cameras. Through the use of analytical self-calibration techniques, accuracy has increased to the point where close-range photogrammetry has become a standard technique for precision (manufacture and service) industrial inspection.

I ts primary applications are industrial, manufacturing, and facilities management; it therefore supports the import and export of data in standard CAD (computer aided design) formats, so that the photogrammetrically produced measurements and models can be easily used for design or analysis. An example of such a system is PhotoM odeler, a product of EOS system [9]. PhotoM odeler accepts photos from both un-calibrated and calibrated cameras. The system defines object in the scene by combinations of geometric primitives, such as cylinders or planes, and operator specifies point or edges to be used in the solution. The models produced can be exported in standard CAD or graphics formats.

\subsection{PHOTOGRAMMETRY CONSISTENT WITH PHOTOMODELER SOFT WARE}

The images can be saved in almost any popular bitmapped format for use with PhotoModeler, including J PEG, TIFF, BMP, PNG, as well as Photoshop and PICT for Macintosh users. To make a 3D model, photographs generated from traditional $35 \mathrm{~mm}$ film prints, digital cameras, as well as video images can be used. EOS System recommends that at least three photos are taken to capture all the important aspects of the object, and that the areas of the images cover overlap each other. The points are then correlated from one 'references' photo with 
corresponding points on the other photos. Close-ups and wide shots can be combined in a project, and new details can be added at any time. DeChant [14] described that by using close-range photogrammetry instead of taking traditional contact measurements, photos were converted into AutoCAD models using PhotoM odeler Pro from EOS System, I nc., Vancouver, British Columbia. Unlike competing programs, PhotoModeler requires no subordinate measurements so there is no need for a survey crew to validate discrete reference locations. The CAD models derived from the photos were highly accurate, and no rework was required when the new ductwork was installed.

Figure 1 shows the flowchart of the main steps of photogrammetry consistent with Photomodeler Pro, one of the software programs used in this research. The following sections of the paper discuss each individually. The flowchart is a closed loop since lessons learned in each application lead to improvements in later applications.

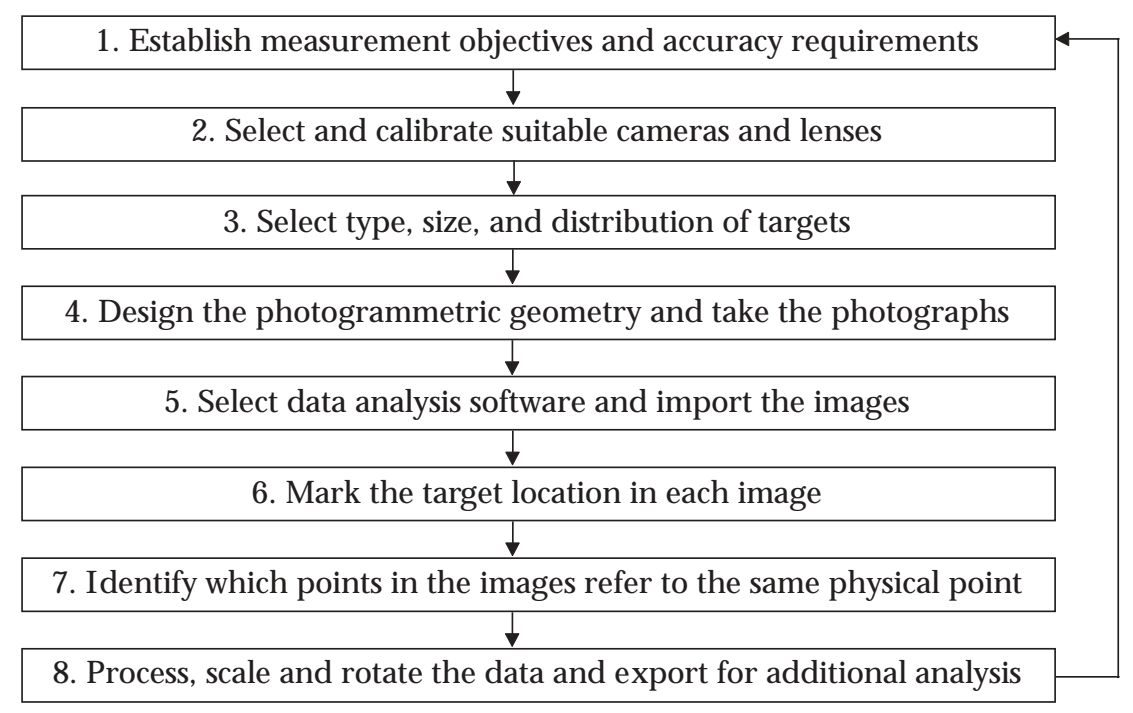

Figure 1 Steps of photogrammetry

\subsection{Establish Measurement $O$ bjectives and Accuracy Requirements}

Establishing the specific measurements of objectives and accuracy requirements is important for selecting proper test methods for the project. A good estimate of the required measurement accuracy for each photogrammetry project is important, avoiding both under- and over-estimating the requirements. Underestimation can lead to unacceptable or unreliable measurements. Over-estimation can waste time and resources because the cost versus accuracy relationships is one of diminishing returns at higher accuracy levels. Photogrammetric accuracy 
specified in parts per thousand of the largest dimension of the structure can vary by more than three orders of magnitude depending on the method and equipment used, ranging from 1:1 000000 with large format film cameras to under 1:1000 with low-resolution consumer cameras and manual feature-marking in images [15].

\subsection{Select and Calibrate Suitable Cameras and L enses}

Modern close-range photogrammetry uses digital cameras almost exclusively rather than traditional film or analog (for video) equipment because of several advantages, including:

(i) the images are immediately available for computer analysis (using removable storage media or cable connection);

(ii) the photogrammetrist can take many extra pictures at the test site at no additional cost using different camera and lighting setting and select the best images later for the analysis; and

(iii) the measurement accuracy can be higher than for standard 35- $\mathrm{mm}$ film, which can shift relative to the camera lens. Also, image transmissions (for video) are of higher quality when using digital data lines.

There are also some disadvantages of digital cameras compared to film or analog (for video) equipment, which include;

(i) higher prices (at least three times higher than comparable film cameras), but these are fully recovered by eliminating film and developing cost,

(ii) maximum image resolution capability is still achieved by medium- or largeformat film cameras designed and calibrated for photogrammetry, and

(iii) cable-length limitations of digital video systems, which is typically less than $10 \mathrm{~m}$ without increased noise.

Tsatsakis [16] defined two types of cameras for photogrammetry:

(i) Metric: where calibration is performed with expensive equipment (in which interior parameters and the mathematical models for lens distortion are well defined).

(ii) Non-metric: where analytical models compensate the systematic co-ordinate errors applied directly to the photogrammetric projective equations (self calibration).

There are many models of consumer, scientific and professional digital cameras on the market, many with similar specifications. The camera used for this project is from Nikon Corporation's Digital Compact Cameras Cool Pix Series. Accurate photogrammetry requires precise knowledge of the optical characteristics of a 
camera. The physical properties of a camera, such as the focal length and lens distortion are known as the 'camera parameters'. The process of measuring these properties is called camera calibration [17]. PhotoM odeler Pro version software contains a simple procedure for computing internal camera parameters by analyzing the photographs of a grid of targets pattern. Please refer to PhotoModeler manual [18] for camera calibration procedure.

\subsection{Select Type, Size and Distribution of Targets}

Photogrammetry achieves the best accuracy using high-contrast, solid-coloured circles as targets. Targets can be light-coloured or dark-coloured on light background, the former being more common. Targets cover each part of the structure with enough density to define its shape, usually without being placed at specific locations, through there can be advantages in placing some targets at known coordinates. Photogrammetry can measure the 3D shape of a structure at each instant of time with projected dots.

\subsection{Design the Photogrammetric Geometry and Take the Photographs}

Designing the photogrammetric geometry involves selecting an adequate number and distribution of camera potions. A general guideline is to place cameras at convergent viewing angles, in both the horizontal and vertical directions if possible, at about $70^{\circ}$ to $90^{\circ}$ angular separation, plus or minus $30^{\circ}$. Each point of interest on the object must appear in at least two photographs for 3D determination, although four or more photographs are preferred for improved least-square accuracy and reliability. With knowledge of at least on other constraint, such as knowing that all object points lie on a plane, photogrammetry can also use a single camera location. This capability is particularly useful for real-time measurements to reduce computational requirements.

Generally, the best way to take the photographs is using a small aperture setting ( $f / 8$ or higher) to obtain good depth of field (i.e., the depth in the object that is simultaneously in focus) while minimizing, or even eliminating, focusing requirements. Using a small aperture and focusing the camera to optimise depth of field, it is possible to simultaneously focus all objects in the picture from a short distance in front of the camera out to the horizon. H owever, using a small aperture requires a slower shutter speed or brighter illumination to obtain adequate image exposure. Most consumer digital cameras have zoom lenses, and it is important to be sure that they are set properly. Normally minimum or maximum zoom setting (focal length) and infinity focus are used to simplify the process and improve repeatability. Any changes in the zoom or focus setting require new camera calibration. The following section discusses about the remaining eight steps by testing on the case study of L arkin Mosque Car Parking Project, J ohor, Malaysia. 


\subsection{PROCESS OF DEVELOPING 3D MODEL FROM IMAGES}

Following sub-sections discuss the process for developing the 3D Model from digital images by using PhotoM odeler software on the case study project.

\subsection{Select Data Analysis Software and I mport the I mages}

With rapidly increasing capabilities of digital cameras and personal computers in recent years, some photogrammetry softwares are made available in the market. These products can analyse images from any source. Photomodeler Pro, one of the consumer software products has been used successfully throughout this research.

First, the images are transferred from the cameras to the computer by using USB flash drive. Before pictures can be transferred (copied) to computer, the supplied software must be installed. Then the Photomodeler software is started, and the images are selected and imported into the program for analysis. Next, the user associates each image with its specific, previously calibrated camera. This allows the proper internal camera parameters, obtained from calibration, to be used with each image.

\subsection{Mark the Target L ocations in Each I mage}

Data analysis begins by marking the locations of the targets in the images. The xy coordinates of the centroid of each elliptical target, projected while dots in this case, must be marked as accurately as possible in each image. An important aspect of precision photogrammetry is the availability of sub-pixel interpolation algorithms that locate the centre of solid-coloured ellipses to an accuracy of onetenth of a pixel or less [19]. The 3D spatial measurement precision obtained with photogrammetry is directly related to this sub-pixel interpolation factor.

Photomodeler contains a robust sub-pixel marking tool for circulate targets. I ndividual targets are marked by clicking them with the mouse, or all targets in a rectangular region of the image can be selected and marked collectively. The latter approach is called automatic marking or auto-marking and although it is not entirely automatic, it greatly simplifies sub-pixel target marking in projects with large numbers of points, such as in dot-projection tests.

\subsection{I dentify Points in the I mages R efers to the Same Physical Point}

The second step of the data analysis is to match the marked points in one image with their corresponding points in the other images. This process is called referencing the points. When a point is initially marked on an image, it is assigned a unique identification number. When a marked point on one image is referenced 
to a marked point on another image, the software reassigns the same identification number to both points indicating they are the same physical location on the structure. In the beginning of the data analysis, the user must perform this referencing operation manually until a certain minimum number of points (at least six) are referenced on all photos, at which time the user processes the data.

When these calculations are completed, the user returns to the referencing phase. At this point, automatic helper tools are available to speed up the process. These tools appear as a result of the initial processing of the data, which yields the spatial location and orientation angles of the cameras. Now the images are said to be oriented. In a typical Photomodeler project without control points specially points with known coordinates, the camera locations and orientations calculated above are relative quantities with respect to the camera. At this point, it is a good idea to verify that the software positioned the cameras properly, which can be checked easily in the graphical 3D viewer available in photomodeler. The viewer shows small camera icons at their locations and orientations relative to targets with calculated 3D coordinates, displayed as small dots. Controls are available to rotate or resize the 3D graphic for better viewing as shown in Figure 2.

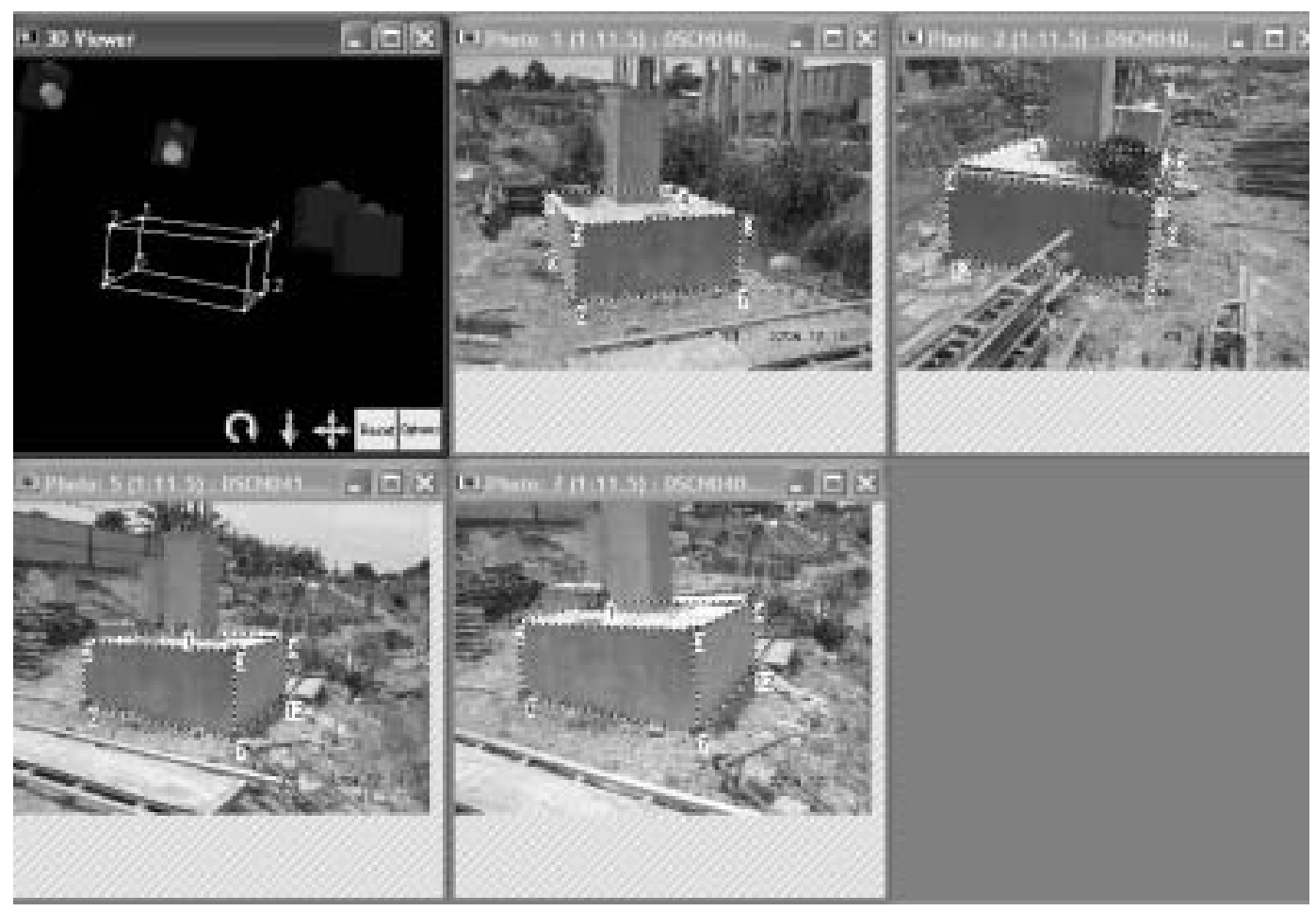

Figure 2 Marking on reference points and 3D viewer with camera icon of the construction of pile cap 


\subsection{Process Scale, and R otate the Data and Export for Additional Analysis}

The third and final step of the data analysis is to "process" the data using the bundle-adjustment algorithm. In the technical literature, several variations of the bundle adjustment method appear, with different user options and levels of sophistication [20]. As discussed in the proceeding section, the data are processed initially after referencing at least six points in each image. This orients the images. Then the user returnsto referencing (and marking, if necessary) additional points. I $t$ is better not to reference all remaining points at this time, but stop after adding some additional points and re-processing the data.

The bundle adjustment al gorithm does two things simultaneously: a) computes the spatial locations and orientation angles of each camera, and b) computes the 3D coordinates of all referenced points and estimates their measurements precision. Bundle adjustment is al ways an iterative solution (since the underlying calculation is non-linear), and hence the calculations continue until a specified consistency or maximum number of iterations occurs. If the object points are distributed on the structure and the photographs are at suitable angles, the bundle adjustment will usually run successfully. I n many cases, camera self-calibration or field-calibration are added to the bundle adjustment equations during the data processing to improve the internal consistency of the solution and the accuracy of the point coordinates.

The processing is done and the coordinates of all targets are successfully determined using successive bundle adjustment calculations. The result in Figure 2 shows the 3D static shape of the pile cap. Following each bundle adjustment, the camera locations and orientations are displayed in the photomodeler 3D viewer and tables in PhotoModeler display the information about: a) the photo table; b) the point table; $c$ ) the edge table; $d$ ) the curve table; e) the surface table; and f) the cylinder table. The cameras al ways appear to be in their proper positions and orientations, adding confidence that the software is working properly. The 3D model created using photomodeler controls all options for exporting all the 3D model data for use in external CAD, graphics, animation and rendering packages. Tables are created similar to spreadsheets with rows and columns of data and export the contents of the currently visible table data contents to an ASCII text file. I $n$ this research, the point table is integrated into the database and compared with database developed by AutoCAD's value and the percentage of the progress will be calculated and the actual physical progress bar chart is developed. Figure 3 shows the point table with coordinate values of different points for the structure and overview of the described points for translate, scale and rotation. 


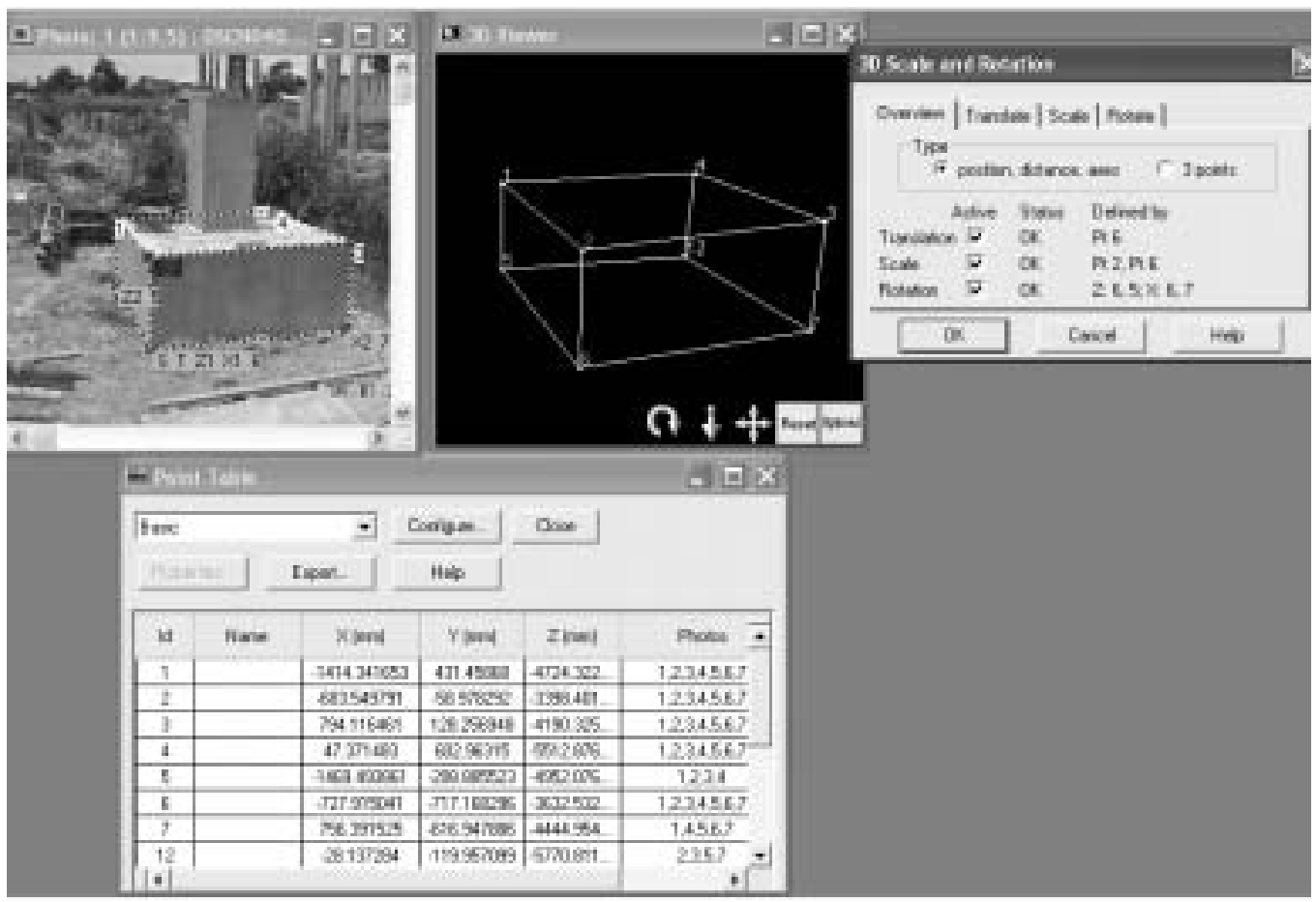

Figure 3 Point table with coordinate values and orientation

\subsection{DISCUSSION}

This paper has described basic method for photogrammetry orientation, the task of which is to derive the object space coordinates from 2D images. The process of developing the 3D Model from 2D digital images is demonstrated by the applications of PhotoM odeler software on a case study project. The results of case study project will help to automate the whole process of construction project. The developed 3D Model is used for developing an automated system for monitoring the project, which is under development at CTMC, UTM, Skudai. The derived 3D coordinate values are stored into database to simulate with AutoCAD drawings to update the project progress. In this regard, this article discussed in detail the process to develop 3D Model 2D digital image and extract the 3D coordinates values by using the newly developed PhotoM odeler software from the Canadian company EOS System. PhotoM odeler is state of the art because it uses innovative technology to allow users to create 3D models. The fact that it does this in an easy-to-use, affordable environment is important because it means that technology is accessi ble to everyday user. 


\subsection{CONCLUSION}

The methodology for developing the 3D Model from digital images and to compute the 3D coordinate values from these has been successfully achieved. These coordinate values are stored into the database. The simulation of these coordinate values with the 3D CAD drawings and calculation of the actual physical progress is out of scope for this paper.

The application of photogrammetry methods in project monitoring and control has been successfully demonstrated in the case study for Larkin Mosque Car Park project. I nitial results indicate that the use of the off-the-shelf digital camera and software technologies are able to provide an accurate method to develop the 3D model and extract the 3D co-ordinate values from digital images. The lesson learned through this will be incorporated with the current research to develop construction monitoring, and automatic evaluation of the physical progress report.

\subsection{ACKNOWLEDGEMENTS}

The authors wish to acknowledge the contributions and support for this study by the Ministry of Science, Technology and I nnovation (MOSTI), Malaysia, for the grant under the research grant number (Vote no. 74107) and Johor Bahru City Council for their cooperation. The permission provided by the contracting organisation for access to construction site is duely acknowledged. Special thanks are also due to I r. Dr. Mahmood Tahir for his valuable assistance.

\section{REFEREN CES}

[1] Memon, Z. A., M. Z. Abd Majid, and M. Mustaffar. 2004. Utilization of Photogrammetry Techniques to Digitalize the Construction Site Progress. International Conference on Construction I nformation Technology (I NCITE)-2004. 18-21 February 2004. Langkawi, Malaysia.

[2] Jervis, B. M. and P. Levin. 1988. Construction Law Principles and Practice. New York, USA: McGraw-Hill.

[3] Rubin, R. A., S. D. Guy, A. C. Maevis, and V. Fair-weather. 1983. Construction Claims, A nalysis P resentation and D ef ense. New York, USA: Van Nostrand Reinhold.

[4] Abd.Majid, M. Z., Z. A. Memon, and M. Mustaffar. 2004. Conceptual Digital Monitoring Model for Evaluating the Progress of Work. Proceedings of $4^{\text {th }}$ Conference of Construction Applications of Virtual Reality (CONVR) 2004. 13-15 September 2004. ADETTI/I SCTE, Lisbon, Portugal. 11-16.

[5] H siek, Y. 1996. Site City: A Semi Automated Site Modeling System. I E E E . 1063-6919/96: 499506.

[6] Streilein, A. and U. Hirschberg. 1995. Integration of Digital Photogrammetry and CAAD: Constraint-Based Modeling and Semi-Automatic Measurement. Proceedings of I nternational Conference on CAAD Future. September, Singapore.

[7] Streilein, A. 1996. Utilization of CAD Models for the Object Oriented Measurement of Industrial and Architectural Objects. International Archives of P hotogrammetry and R emote Sensing. XXI (B5): 548-553. 
[8] Gomes, J . M. G., W. da S. Prado, H. Erwes, and G. D. Koatz. 1999. A Photogrammetric Project in Brazil: The Use of the Photomodeler Software. http://cipa.icomos.org/fileadmin/papers/ onlinda/99C3B.pdf (Accessed on 12/06/2004).

[9] Mikhail, E. M., S. J. Bethel, and J. C. McGlone. 2001. Introduction to M odern P hotogrammetry. New York, USA: J ohn Wiley \& Sons, I nc.

[10] Greco, J . 2001. 3D I nput with PhotoM odeler from Eos System. Cadence; P ro Q uest Computing. http://www.findarticles.com/p/articles/mi_hb3220/is_200110/ai_n7897270 (Accessed on 20/7/2005).

[11] Atkinson, K. B. 2001. Close-R ange P hotogrammetry and M achine Vision. Scotland, UK: Whittles Publishing Company.

[12] El-Hakim, S. F. 2001. Vidoemetrics and Optical Methods for 3D Shape Measurement. SPIE Proceedings. N o. 4309.

[13] Boochs, F., A. Gehrhoff, and M. Neifer. 2000. An Object-Oriented Stereo System for 3DMeasurement. IAPR S. XXXIII : 1-8.

[14] DeChant, I. 1999. CAD Models Made from Photos Reduce Furnace Down-Time. I ndustrial Headings. Professional Surveyor Magazine. 19(3). http://www.profsurv.com/newpsm/ archive. php?issue $=30$ \&article $=407$ (Accessed on 21/6/2005).

[15] Pappa, R. S., T. W. J ones, J. T. Black, A. Walford, S. Robson, and M. R. Shortis. 2002. Photogrammetry Methodology Development for Goassamer Spacecraft Structures: N ational Aeronautics and Space Administration NASA/T M-2002-211739.

[16] Tsatskis, I ., E. Dayafas, V. Loumos, and G. Cambourakis. 1995. Using L ow Cost Video Cameras in Automation a Close-Range Photogrammetry Approach. IE E E . 95T H 8081.

[17] Fryer, J. G. 2001. Camera Calibration, Close-Range Photogrammetry and Machine Vision. In K. B. Atkinson (Ed.). Scotland, UK: Whittles Publishing.

[18] PhotoModeler Pro. 2004. Version 5. U ser Manual Eos System I nc. 1991-2004.

[19] West, G. A. W. and T. A. Clarke. 1990. A Survey and Examination of Sub Pixel Measurement Techniques. SPIE Proceedings. 1395: 456-463.

[20] Granshaw, S. I. 1980. Bundle Adjustment Methods in Engineering Photogrammetry. P hotogrammetric R ecord. 10 (56):181-207. 